\title{
An Algorithm to detect balancing of iterated line sigraph
}

\author{
Deepa Sinha ${ }^{1 *}$ and Anshu Sethi ${ }^{2}$
}

\author{
*Correspondence: \\ deepa_sinha2001@yahoo.com \\ ${ }^{1}$ South Asian University Akbar \\ Bhawan, Chanakyapuri, \\ New Delhi 110 021, India \\ Full list of author information \\ is available at the end of the \\ article
}

\begin{abstract}
A signed graph (or sigraph in short) $S$ is a graph $G$ in which each edge $x$ carries a value $s(x) \in\{+1,-1\}$ called its sign denoted specially as $S=(G, s)$. Given a sigraph $S, H=$ $L(S)$ called the line sigraph of $S$ is that sigraph in which edges of $S$ are represented as vertices, two of these vertices are defined to be adjacent whenever the corresponding edges in $S$ have a vertex in common and any such edge ef is defined to be negative whenever both $e$ and $f$ are negative edges in $S$. Here $S$ is called root sigraph of $H$. Iterated signed line graphs $L^{k}(S)=L\left(L^{k-1}(S)\right), k \in \mathbb{N}, S:=L^{0}(S)$ is defined similarly. In this paper, we give an algorithm to obtain iterated line sigraph and detect for which value of ' $k$ ' it is balanced and determine its complexity. In the end we will propose a technique that will use adjacency matrix of $S$ and adjacency matrix of $L^{k}(S)$ which is balanced for some ' $k$ ' as a parameter to encrypt a network and forward the data in the form of balanced $L^{k}(S)$ and will decrypt it by applying inverse matrix operations.
\end{abstract}

Keywords: Sigraph, Line sigraph, Balanced sigraph, Iterated line sigraph, Negative section, Network, Encryption and decryption

Mathematics Subject Classification: Primary 05C 22, Secondary 05C 75, 05 C 85

\section{Background}

For standard terminology and notation in graph theory we refer to Harary (1969), West (1996) and Zaslavsky $(1981,1982)$ for sigraphs and Cormen et al. (2011) and Golumbic (2004) for algorithms. Throughout the text, we consider finite, undirected graph with no loops or multiple edges. By an $(n, e)$ graph $G$ we mean a graph having $n$ vertices and $e$ edges; $n$ is called the order and $e$ is called the size of G. In computer science domain, any graph $G$ is observed as a network by computer scientists where vertices are taken to be nodes and edges to be taken as links.

A signed graph (or sigraph in short) Zaslavsky (1982) is an ordered pair $S=\left(S^{u}, \sigma\right)$, where $S^{u}=(V, E)$ is a graph called the underlying graph of $S$ and $\sigma: E \rightarrow\{+,-\}$ is a function from the edge set $E$ of $S^{u}$ into the set $\{+,-\}$, called the signature (or sign in short) of $S$.

For a sigraph $S$, Behzad and Chartrand (1969) define its line sigraph, $L(S)$ as the sigraph in which the edges of $S$ are represented as vertices, two of these vertices are defined adjacent whenever the corresponding edges in $S$ have a vertex in common, any such edge $e f$ is defined to be negative whenever both $e$ and $f$ are negative edges in $S$. A

\section{Springer}

(c) 2015 Sinha and Sethi. This article is distributed under the terms of the Creative Commons Attribution 4.0 International License (http://creativecommons.org/licenses/by/4.0/), which permits unrestricted use, distribution, and reproduction in any medium, provided you give appropriate credit to the original author(s) and the source, provide a link to the Creative Commons license, and indicate if changes were made. 
given sigraph $S$ is a linesigraph if it is isomorphic to the linesigraph $L(T)$ of a sigraph $T$. Here $T$ is called the line root of $S$. A sigraph $S$ and its line sigraph $L(S)$ is shown in Fig. 1.

A sigraph $S$ is sign-compatible Sinha (2005); Sinha and Sethi 2015) if there exists a marking $\mu$ of its vertices such that the end vertices of every negative edge receive '-' signs in $\mu$ and no positive edge in $S$ has both of its ends assigned '-' sign in $\mu$. In other words, a sigraph is sign-compatible if and only if its vertices can be partitioned into two subsets $V_{1}$ and $V_{2}$ such that the all-negative subsigraph of $S$ is precisely the subsigraph induced by exactly one of the subsets $V_{1}$ and $V_{2}$. Every line sigraph is sign-compatible. However, not every sign-compatible sigraph need be line sigraph.

An adjacency matrix for a network with $n$ vertices and no parallel edges is an $n \times n$ symmetric matrix such that

$$
a_{i j}= \begin{cases}1 & \text { if }(i, j) \text { is a solid line } \\ -1 & \text { if }(i, j) \text { is a dotted line } \\ 0 & \text { if }(i=j)\end{cases}
$$

A cycle in a sigraph $S$ is said to be positive if the product of the signs of its edges is positive or, equivalently, if the number of negative edges in it is even. A cycle which is not positive is said to be negative. A sigraph is said to be balanced if every cycle in it is positive (Harary 1953; Cartwright and Harary 1956; Acharya and Acharya 1986). The following characterization of balanced sigraphs is well known:

Theorem 1 (Harary and Kabell 1980) A sigraph is balanced if and only if there exists a partition of its vertex set into two subsets, one of them possibly empty, such that every positive edge joins two vertices in the same subset and every negative edge joins two vertices from different subsets.

For any positive integer $k$, the $k$ th iterated line sigraph $L^{k}(S)$ of $S$ is defined (see Gill and Patwardhan 1983) recursively as follows:

$L^{0}(S)=S, L^{k}(S)=L\left(L^{k-1}(S)\right)$.

A sigraph $S$ and its iterated line sigraph $L^{k}(S)$ is shown in Fig. 2.

By a negative section (Gill and Patwardhan 1981) of a subgraph $S^{\prime}$ of a sigraph $S$ we mean a maximal edge-induced subgraph in $S^{\prime}$ consisting of only the negative edges of $S$; in particular, a negative section in a cycle of $S$ is essentially a maximal all-negative path
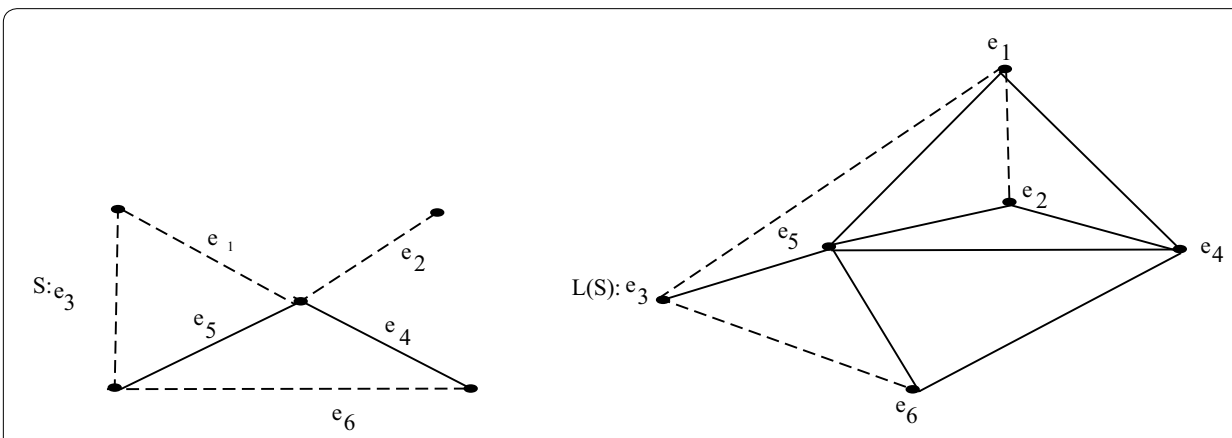

Fig. 1 Example showing sigraph and its line sigraph 


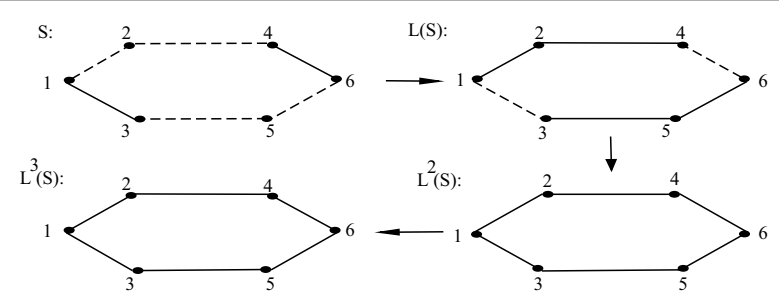

Fig. 2 Example showing sigraph and its line sigraph upto 3 iterations

in the cycle or the whole cycle itself. Thus, a cycle is positive if and only if it has an even number of negative sections of odd length.

In this paper, we are going to introduce a new method for encoding and decoding of data using network as sigraphs and basic properties of matrices. For the purpose of network security, adjacency matrix of $S$ will be considered as basis of information which is to encrypted to adjacency matrix of balanced $L^{k}(S)$ for some 'k' to assure confidentiality, integrity and authentication of transmitted data.

The following result gives a characterization of sigraphs whose line sigraph $L(S)$ is balanced:

Theorem 2 (Sinha 2005) For any sigraph S, L(S) is balanced if and only if the following conditions hold on $S$ :

1. For any cycle $Z$ in $S$;

(a) If $Z$ is all negative then $Z$ is of even length;

(b) If $Z$ negative sections of non-zero even length inis heterogenous then there is an even number of $Z$;

2. For any vertex $v$ in $S$, if the degree exceeds two then there is at most one negative edge incident at $v$.

\section{Balanced iterated signed line graphs}

In this section, we extend Theorem 2 to any iterated line sigraph $L^{k}(S), k \in \mathbb{N}$.

Theorem 3 (Sinha 2005; Sinha and Acharya 2015) For any sigraph S, and for any positive integer $k, L^{k}(S)$ is balanced if and only if the following conditions are satisfied by $S$ :

1. For any cycle $Z$ in $S$;

(a) If $Z$ is all negative then $Z$ is of even length;

(b) If $Z$ is heterogenous then the number of negative sections of odd(even) length greater than $k$ is even if $k$ is even(odd); and

2. For any vertex $v$ in $S$, if $d(v)>2$ then $d^{-}(v)<3$, and if $d^{-}(v)=2$, then length of any negative section through $v$ is at most $k$.

A sigraph $S$ and its iterated line sigraph such that $L^{1}(S)$ is not balanced and $L^{2}(S)$ is balanced is shown in Fig. 3. 


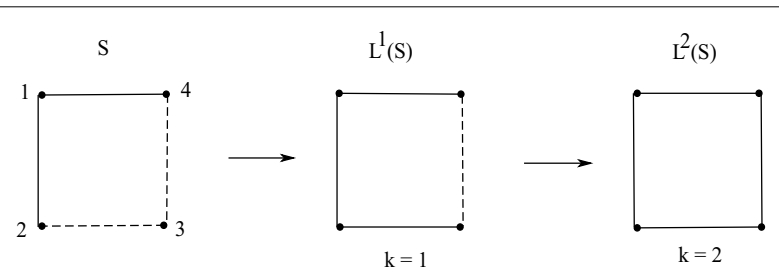

Fig. 3 For a sigraph $S, L^{1}(S)$ is not balanced and $L^{2}(S)$ is balanced

Numerical interpretation of above characterization

Following is the numerical analysis for the sigraphs shown in Fig. 3. The adjacency matrix corresponding to sigraph $S$ is defined as follows:

$$
A(S)=\left[\begin{array}{cccc}
0 & 1 & 0 & 1 \\
1 & 0 & -1 & 0 \\
0 & -1 & 0 & -1 \\
1 & 0 & -1 & 0
\end{array}\right]
$$

Following procedure is implemented to obtain an iterated line sigraph from a given sigraph and check whether this iterated line sigraph is balanced or not:

Enter the number of nodes, i.e., $n$. Input $n \times n$ adjacency matrix with respect to given sigraph. The adjacency matrix takes the entries as 0,1 and -1 for no edge, positive edge and negative edge respectively. Now we have to find the line sigraph of this sigraph Sinha and Sethi (2015). For this, first we populate EdgeList (list of all edges in the given sigraph) by assigning each edge an index. We then search for non-zero entries in adjacency matrix and then corresponding to each such entry, say $(i, j)$ th entry, we assign the edge number as $1,2,3, \ldots$

Then for each edge we check adjacent edges from the EdgeList prepared and see if they have the common vertex. The sign of the vertex depends on the sign of edge $(i, j)$ th in $S$. If edge $(i, j)$ is positive then corresponding vertex would be positive otherwise it would be negative. This way new matrix of $L(S)$ is computed.

Since we have to compute $L^{k}(S)$, it means we have to repeat the above conversion of converting sigraph to its line sigraph ' $k$ ' times such that number of edges of first iterated line sigraph now becomes number of vertices for the second iterated line sigraph and output matrix of first iterated line sigraph is now the input matrix for the required second iterated line sigraph and so on.

Similarly, $L^{k}(S)$ is computed.

To check 1(a) condition, i.e. if cycle is negative, we will find all cycles in the given sigraph. For each cycle we calculate the length of the path and count all negative entries corresponding to this path. If path length is equal to half of the negative values, it means that cycle is homogenous i.e. it contains all the negative edges otherwise heterogenous. If cycle is homogeneous, then count must be even otherwise we terminate the procedure and say $L^{k}(S)$ is not balanced.

For the adjacency matrix of $S$, length of the path 1-2-3-4 of cycle is 4 and half count of all negative entries is $=4 / 2$ i.e. 2 . Since length of path of cycle does not match the number of negative entries, therefore, cycle is not homogenous and we check the next condition. 
To check 1(b) condition we first find a cycle. Then we search for positive edge in the path. If we found any positive edge it means the cycle is heterogeneous. Now we count1 entries in the path. If it contains only one negative edge proceeded with a positive edge consecutively in the path, it means it has negative section. Now we count -1 entries and check if it is greater than ' $k$ ' and of odd length, we update NbOddNegativeSections (number of negative sections of odd length) by 1 and if it is greater than ' $k$ ' and of even length then we increment $\mathrm{NbEvenNegativeSections} \mathrm{(number} \mathrm{of} \mathrm{negative} \mathrm{sections} \mathrm{of} \mathrm{even} \mathrm{length)}$ by 1 . This way total number of negative sections of odd length and even length greater than ' $k$ ' is calculated. Now we check if this count NbOddNegativeSections is even when ' $k$ ' is even and NbEvenNegativeSections is odd when ' $k$ ' is odd. If both the condition are satisfied we check the next condition otherwise we terminate the procedure.

For the adjacency matrix of $S$, it has 2 negative sections, 1-2-3 of length 2 and 4-5-6 also of length 2 . Take $k=1$ i.e. ' $k$ ' is odd. Count number of negative sections of even length $>k$, which is 2 but according to the theorem this count must be odd. Given condition does not hold true for $k=1$, therefore for given sigraph $S, L^{1}(S)$ is not balanced. Take $k=2$ i.e. ' $k$ ' is even, count number of negative sections of odd length $>k$, which is 0 in our case and is even. Thus, given condition holds true for $k=2$ and we proceed to check the next condition.

To check 2 condition we calculate degree of each vertex by counting non-zero entry in each row and negative degree by counting -1 entries in each row. Then for each vertex we check if degree is $>2$ then negative degree must be $\leq 3$ and if $d^{-}(v)=2$, then we calculate again the length of each negative section incident at $v$ by applying the same procedure as calculated in condition $1(\mathrm{a})$.

For the adjacency matrix of $S$, there exists no vertex where degree $>2$, therefore, given condition is also satisfied for given $S$. Thus, we can say that for given sigraph $S, L^{1}(S)$ is not balanced and $L^{2}(S)$ is balanced.

\section{Algorithm to detect balancing of iterated line sigraph}

The algorithm to detect balancing of line sigraph is based on the characterization given by Acharya and Sinha (2003). To detect balancing of iterated line sigraph $L^{k}(S)$ of $S$, we have to first obtain $L^{k}(S)$ from $S$. Following is the algorithm to $L^{k}(S)$ of a given sigraph $S$ :

\section{Algorithm to convert a sigraph to its iterated line sigraph SigraphtolteratedLinesigraph (vertex, $n, k$ )}

Here vertex is the input matrix and $n$ is the number of vertices of a given sigraph. $k$ denotes number of iterations whose $k$ th iterated line sigraph $L^{k}(S)$ is to be computed.

Step 1. Apply algorithm defined in [23] to obtain $L(S)$ i.e matrix linevertex $[i][j]$.

Step 2. Count non-zero entries in matrix linevertex $[i][j]$. Let this count be $m$.

Step 3. Set $n=m / 2$

$/ /$ number of edges will now become number of vertices for next iteration.

Step 4. Set vertex $[i][j]=$ linevertex $[i][j]$ for $\mathrm{i}=1$ to $\mathrm{n}$ and $\mathrm{j}=1$ to $\mathrm{n}$

// output matrix will now become input matrix for next iteration.

Step 5. Repeat Step 1 to Step 5 for $\ell=2$ to $k$

Step 6. Exit

Note: Since, if we have $n$ number of vertices, then for $k=1$, there are maximum of $n(n-1) / 2$ edges, which in turn becomes number of vertices for the next iteration and 
so on. For $k=2$, the number of vertices will be of order $n^{2}$ and correspondingly number of edges will be of order $n^{4}$ and so on. Thus, $k$ th iteration will have maximum $n^{k}$ number of vertices and $n^{2 k}$ number of edges.

\section{Complexity of computation involved in above algorithm}

In Step 1, we have applied the algorithm defined in Sinha and Sethi (2015) to obtain $L(S)$, Thus complexity of this step $=O\left(n^{2}\right)$.

In Step 5, we have to compute $L^{k}(S)$ and we have to repeat Step1, $k-1$ times, therefore,

Complexity of this step $=n^{k-1} \times O\left(n^{2}\right)=O\left(n^{2(k-1)}\right)$, where $k$ denotes number of iterations.

Hence complexity of computation involved in above algorithm is $O\left(n^{2(k-1)}\right)$, where $n$ is number of vertices in $S$.

\section{Numerical interpretation}

The adjacency matrix corresponding to sigraph $L^{1}(S)$ and $L^{2}(S)$ is defined as follows:

$$
A\left(L^{1}(S)\right)=\left[\begin{array}{cccc}
0 & 1 & 1 & 0 \\
1 & 0 & 0 & 1 \\
1 & 0 & 0 & -1 \\
0 & 1 & -1 & 0
\end{array}\right] \text { and } A\left(L^{2}(S)\right)=\left[\begin{array}{llll}
0 & 1 & 1 & 0 \\
1 & 0 & 0 & 1 \\
1 & 0 & 0 & 1 \\
0 & 1 & 1 & 0
\end{array}\right]
$$

\section{Main algorithm}

Following is the algorithm to detect balancing of an iterated line sigraph:

\section{IteratedLineSigraphBalance (vertices, $\mathbf{n}, \mathbf{k}$ )}

Here vertices is the input matrix and $n$ is the number of vertices of a given sigraph. $k$ denotes number of iterations. NbPositiveEdges, NbNegativeEdges and NbTotalEdges represents total number of positive edges, negative edges and total edges incident to each vertex. 
Step 1. Implement algorithm defined in Section 2.2.1 to obtain $L^{k}(S)$.

Step 2. Repeat Step 2 to Step 4 for Node $\mathrm{i}=1$ to $\mathrm{n}$

Step 3. Set path.push-back(i)

IsNodeInPath $[\mathrm{i}]=$ true

Step 4. Call function FindCycle(n, i, \&path, IsNodeinPath, IsPathEval

uatedforNode, Evaluatepath)

If it returns 0 ,

Print Since condition on $S$ is not satisfied, therefore, $L^{k}(S)$ is not

balanced and Goto Step 15

Step 5. Print Since 1(b) condition is satisfied i.e cycle is heterogenous and there is even number of negative sections of non-zero even length in the cycle, Now we check the 2nd condition

Step 6. Repeat Step 6 to Step 13 for Node $\mathrm{i}=1$ to $\mathrm{n}$

Step 7. Set NbPositiveEdges $=0$

NbNegativeEdges $=0$

NbtotalEdges $=0$

Step 8. Repeat Step 8 to Step 9 for $\mathrm{j}=1$ to $\mathrm{n}$

Step 9. if (vertices $[\mathrm{i}][\mathrm{j}]==1$ ) Set NbPositiveEdges ++

if (vertices $[\mathrm{i}][\mathrm{j}]==-1)$ NbNegativeEdges ++

if $(($ vertices $[\mathrm{i}][\mathrm{j}]==-1) \|(\operatorname{vertices}[\mathrm{i}][\mathrm{j}]==1))$ NbTotalEdges ++

Step 10. Repeat Step 10 to Step 13 for $\mathrm{i}=1$ to $\mathrm{n}$

Step 11. Check if(NbTotalEdges $>2$ )

Check if(NbNegativeEdges $>3$ ), If yes,

Print Since condition is not satisfied, therefore, $L^{k}(S)$ is not balanced and Goto Step 15

Step 12. check if(NbNegativeEdges $==2)$, Set

path.push-back(i)

IsNodeInPath $[\mathrm{i}]=$ true

Step 13. Call function FindCycle(n, i, \&path, IsNodeinPath, IsPathEvaluated forNode, EvaluatePath)

If it returns 0 ,

Print Since second condition on $S$ is not satisfied, therefore, $L^{k}(S)$ is

not balanced and Goto Step 15

Step 14. Print All conditions on $S$ are satisfied, therefore $L^{k}(S)$ is balanced.

Step 15. Exit

Here FindCycle is a function used to find all cycles within a given sigraph from its adjacency matrix. StartNode represents first node from where the cycle start. PathUptilNow is a vector used to represent the position of the node till the vertex is traversed. IsNodeInPath is a boolean varible used to detect whether nodes is already in the path or not. IsPathEvaluatedForNode is also a boolean variable used to find whether nodes for which path is already calculated. Evaluate $=0$ is a function to evaluate path. cur-node represents the current node which is traversed.

Function FindCycle (n, StartNode, Pathuptilnow, IsNodeinPath, IsPathEvaluatedforNode, Evaluate $=0$ ) 
Step 1. Set Cur-Node $=$ Pathuptilnow $\rightarrow \operatorname{back}()$;

Step 2. Repeat Step 2 for $\mathrm{i}=1$ to $\mathrm{n}$

2.1. Check if ((Cur-Node != i) \&\& (vertex [Cur-Node][i] $))$

2.1.1. Check if $((\mathrm{i}==$ StartNode $) \& \&($ PathUptilNow $\rightarrow$ size ()$>2))$

2.1.1.1. // We have found a cycle

if (Evaluate)

Set AllOkay $=$ Evaluate(PathUptilNow);

if (!AllOkay)

return false;

2.2. else if (IsNodeinPath[i] \| IsPathEvaluatedForNode[i])

continue; // Either the node is already in path, or all the cycles for this node have been evaluated. We don't want this node in the cycle.

2.3. // Add the node to the path and continue to find cycles.

Pathuptilnow $\rightarrow$ push-back(i);

IsNodeinPath $[\mathrm{i}]=$ true;

IsAllOkay $=$ FindCycle( n, StartNode, Pathuptilnow, IsNodeinPath,

IsPathEvaluatedForNode, Evaluate);

IsNodeinPath $[\mathrm{i}]=$ false;

Pathuptilnow $\rightarrow$ pop-back () ;

if (!IsAllOkay) return false;

Step 3. Return true

Above function outputs a path of the cycle. Here Function EvaluatePath is used to detect whether cycle is homogenous or heterogenous. If it is heterogenous, then it counts number of negative sections of odd(even) length greater than $k$ is even if $k$ is even(odd) or not. NbEdgesInSection denotes total number of edges in each section. NbNegativeSections represents total number of negative sections. NbEvenNegativeSections and $\mathrm{NbOddNegativeSections}$ denotes number of negative sections of even and odd length respectively.

Function evaluate (path) 
Step 1. Set NbNodesInPath $=$ path $\rightarrow$ size ()

Step 2. Set StartIndex $=-1$ and PositiveEdgePresent $=$ false

Step 3. // Check ' $1(\mathrm{~b})$ ' condition for heterogenous cycle

// Find the first positive edge

Repeat Step 3 to Step 4 for $\mathrm{i}=1$ to NbNodesInPath

Step 4 . Check if (vertices $[(*$ path) $[\mathrm{i} \%$ NbNodesInPath $]][(*$ path $)[(\mathrm{i}+1) \% \mathrm{Nb}$ NodesInPath]] ==1),

If yes, Set

PositiveEdgePresent $=$ true

StartIndex $=\mathrm{i}$

break

Step 5. if (!PositiveEdgePresent)

// The cycle doesn't have any positive edge, which means it has all negative edges

Print Cycle doesn't have positive edges. Size of cycle is =

NbNodesInPath

return (NbNodesInPath $\% 2==0)$

Step 6. Else Set NbNegativeSections $=0$

NbEdgesInSection $=0$

NbEvenNegativeSections $=0$

NbOddNegativeSections $=0$

IsCurrentSectionNegative $=$ false

Step 7. Repeat Step 7 to Step 14 for $\mathrm{i}=$ StartIndex to (StartIndex + NbNodes InPath +1$)$

Step 8. Check if (vertices[(* path) $[\mathrm{i} \%$ NbNodesInPath $]][(*$ path $)[(\mathrm{i}+1) \% \mathrm{Nb}$

NodesInPath] $==-1)$

Check if (!IsCurrentSectionNegative)

If yes, Print Started negative section at (* path)[i \% NbNodesInPath]

and Set NbNegativeSections++

IsCurrentSectionNegative $=$ true

NbEdgesInSection++

Step 9. else if (IsCurrentSectionNegative)

Print Finished negative section at (*path) [i \% NbNodesInPath] of size

NbEdgesInSection

Check if ((NbEdgesInSection \% $2 !=0) \& \&($ NbEdgesInSection $>$ k $))$

if yes, set NbOddNegativeSections++

Check if ((NbEdgesInSection \% $2==0) \& \&($ NbEdgesInSection $>\mathrm{k}))$

If yes, set NbEvenNegativeSections++

Set NbEdgesInSection $=0$

IsCurrentSectionNegative $=$ false

Step 10. Print Total number of negative sections - NbNegativeSections

Total number of negative sections of even length greater than $\mathrm{k}$ -

NbEvenNegativeSections

Total number of negative sections of odd length greater than $\mathrm{k}$ -

NbOddNegativeSections

Step 11. Check if $((\mathrm{k} \% 2==0) \& \&($ NbOddNegativeSections $\% 2==0))$

If yes, return (true)

Step 12. Check if $((\mathrm{k} \% 2 !=0) \& \&($ NbEvenNegativeSections $\% 2==0))$

If yes, return (true)

Step 13. Check if(NbEdgesInSection $>$ k), If yes, return(false)

checking 2nd condition

Step 14. return(false)

\section{Complexity of computation involved in above algorithm}

In Step 1, we compute $L^{k}(S)$ as defined in "Main algorithm", therefore,

Complexity of this step $=O\left(n^{2(k-1)}\right)$. 
In Step 2, each node of the graph is traversed and for each node we have to find cycle i.e. Step 4 is called ' $n$ ' times. For finding cycle, we have to call for function EvaluatePath () where each vertex is again traversed. Thus complexity of this step $=O(n) \times n \times n$ $=O\left(n^{3}\right)$.

For the function EvaluatePath(), in Step 3 and Step 4 we first calculate first positive edge and for this we have to traverse each node of the path, Thus, Complexity of this step $=O(n)$.

In Step 7, for finding number of negative sections and length of each section we repeat Step 7 to Step 12 and it is repeated for each cycle, therefore, Complexity of this step $=$ $O(n) \times n=O\left(n^{2}\right)$.

For checking the 2 condition we calculate positive, negative and total edges incident to each vertex, for this, we follow Step 6 to Step 9 in the main algorithm and for this we traverse again $n \times n$ matrix, Thus, complexity of this step $=O\left(n^{2}\right)$.

In Step 10 to Step 13, we check total degree and negative degree of each vertex, therefore, Complexity of this step $=O(n)$.

In Step 13, we call the function FindCycle(), such that whole procedure is again repeated, therefore, Complexity of this step $=O(n) \times n \times n=O\left(n^{3}\right)$.

Total complexity $=O\left(n^{2(k-1)}\right)+O\left(n^{3}\right)+O(n)+O\left(n^{2}\right)+O\left(n^{2}\right)+O(n)+O\left(n^{3}\right)=$ $O\left(n^{3}+n^{2(k-1)}\right)$.

Note: Our main aim is to only detect balancing of iterated line sigraph, therefore, complexity to obtain iterated line sigraphs can be neglected.

Thus Total complexity for detecting balancing of iterated line sigraph $=O\left(n^{3}\right)+O(n)$ $+O\left(n^{2}\right)+O\left(n^{2}\right)+O(n)+O\left(n^{3}\right)=O\left(n^{3}\right)$.

Hence complexity of computation involved in above algorithm is $O\left(n^{3}\right)$, where $n$ is number of vertices in $S$.

\section{Correctness of the above algorithm}

An algorithm is said to be correct if for every input data that satisfies some conditionscalled the precondition of the algorithm, the output data satisfy a certain predefined condition-called the post condition of the algorithm. A graph algorithm depends upon number of vertices and edges in a graph and inter/intra relationship between these two features. Here, we will discuss an approach based on the adjacency matrices of the given sigraph to prove or disprove the correctness of the above proposed algorithm.

Example 1 Consider the sigraphs shown in Fig. 4.

The adjacency matrix corresponding to sigraph $S_{1}$ and $S_{2}$ is defined as follows:
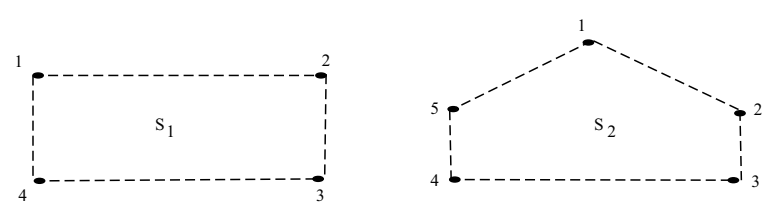

Fig. 4 Example showing homogeneous cycle of odd and even length 


$$
A\left(S_{1}\right)=\left[\begin{array}{cccc}
0 & -1 & 0 & -1 \\
-1 & 0 & -1 & 0 \\
0 & -1 & 0 & -1 \\
-1 & 0 & -1 & 0
\end{array}\right] \quad \text { and } \quad A\left(S_{2}\right)=\left[\begin{array}{ccccc}
0 & -1 & 0 & 0 & -1 \\
-1 & 0 & -1 & 0 & 0 \\
0 & -1 & 0 & -1 & 0 \\
0 & 0 & -1 & 0 & -1 \\
-1 & 0 & 0 & -1 & 0
\end{array}\right]
$$

In Step 4, for $S_{1}$, we call for the function FindCycle() to find all cycles starting with initial node $\mathrm{i}=1$. $S_{1}$ contain cycle 1-2-3-4-1 with path length 4(Path size) and count of negative edges(NbNodesInPath) as 4 . Both the counts are same, therefore, cycle is homogenous and we have to check if this count is odd or even. If it is odd, then process is terminated after Step 4 in $O\left(n^{3}\right)$ ) steps otherwise next condition is checked. Similarly, for $S_{2}$, there exists cycle 1-2-3-4-5-1 with path length 5(Path size) and count of negative edges(NbNodesInPath) as 5 . Both the counts are same, therefore, cycle is homogenous and we have to check if this count is odd or even. Since this count is odd, then process is terminated after Step 4 in $O\left(n^{3}\right)$ ) steps.

Note: For any sigraph to detect its balancing, we have to find cycle and complexity of finding cycle is $O\left(n^{3}\right)$, therefore, whole process must be implemented in atleast $O\left(n^{3}\right)$ steps.

Example 2 Consider another sigraphs as shown in Fig. 5.

The adjacency matrix corresponding to sigraph $S$ and $S_{1}$ is defined as follows:

$$
A(S)=\left[\begin{array}{cccc}
0 & -1 & 0 & -1 \\
-1 & 0 & -1 & 0 \\
0 & -1 & 0 & -1 \\
-1 & 0 & -1 & 0
\end{array}\right] \text { and } A\left(S_{1}\right)=\left[\begin{array}{ccccc}
0 & -1 & 0 & 0 & -1 \\
-1 & 0 & -1 & 0 & 0 \\
0 & -1 & 0 & -1 & 0 \\
0 & 0 & -1 & 0 & -1 \\
-1 & 0 & 0 & -1 & 0
\end{array}\right]
$$

Consider a sigraph $S$ as shown in Fig. 5. It has 2 negative sections, 1-2-3 of length 2 and 4-5-6 also of length 2 . Take $k=1$ i.e. $k$ is odd. Now we have to count number of negative sections of even length $>k$, which is 2 but according to the theorem this count must be odd. Since condition does not hold true for $k=1$, therefore for given sigraph $S, L^{1}(S)$ is not balanced and process is terminated at Step 4 of main algorithm and implemented in $O\left(n^{3}\right)$ steps. If $k=2$ i.e. $k$ is even, then we have to count number of negative sections of odd length $>k$, which is 0 in our case and is even. Therefore, condition holds true for $k$ $=2$ and we proceed to check the next condition. Since there does not exists any vertex with NbTotalEdges $>2$, therefore, given condition is also satisfied and process is again terminated in $O\left(n^{3}\right)$ steps.

Similarly, if we take sigraph $S_{1}$, we have 2 negative sections, 5-1 of length 1 and 2-3-4 of length 2 . Now it can be easily verified that for $k=1$ and $k=2$, all conditions holds true.
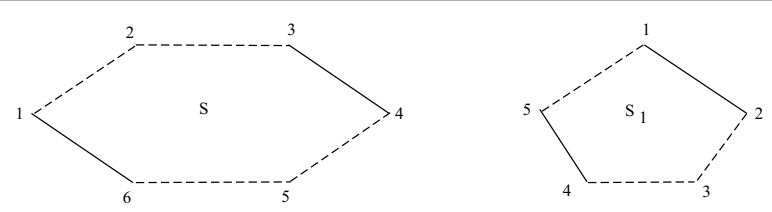

Fig. 5 Illustration of another example 


\section{Conclusion and scope}

In this paper, we have given an algorithmic approach to obtain iterated line sigraphs of a given sigraph and detect whether it is balanced or not in $O\left(n^{3}\right)$ steps. In this method, data is taken in the form of adjacency matrix with entries as 0,1 and -1 . Now since any matrix can be represented in the form of a network and vice-versa, we can apply encryption and decryption mechanism for a network through matrices. Algorithm is already defined to obtain a line sigraph from a given sigraph Sinha and Sethi (2015) and if we have algorithm to obtain line root sigraph of given sigraph, therefore, we can apply encryption and decryption algorithms with the additional condition that $L^{k}(S)$ is balanced for some ' $\mathrm{k}$ ' to a network. There exists several techniques on encryption and decryption, but we have developed a new technique in which weak and strong relationships among nodes in a balanced network can be defined. For the purpose of security, we have used asymmetric key cryptography.

\section{System model}

Asymmetric cryptography is used in this model. Two different keys, a secret key $d$ and a public key $e$ are defined. The public key $e$ is used for encryption i.e. for converting adjacency matrix of $S$ to adjacency matrix of $L^{k}(S)$ which is balanced for some 'k'. Since we have a unique method for encryption, therefore, it can be published. Further, the secret private key $d$ is used for decryption of adjacency matrix of $L^{k}(S)$ to adjacency matrix of $S$. Since we have many line root sigraphs of a given sigraph and we have to restrict our networks to obtain a unique line root sigraph, therefore, private key is used. Also, if labelling of vertices can be done by Lehot (1974), then only we obtain unique line root sigraph. The following model is used as an application to above algorithms.

The model used as an application to above algorithms can be referred in Fig. 6.

\section{Algorithms for system model}

Apart from algorithms defined in "Algorithm to convert a sigraph to its iterated line sigraphSigraphtoIteratedLinesigraph (vertex, n, k)" and "Main algorithm", following are the algorithms that will be used in encryption and decryption mechanism.

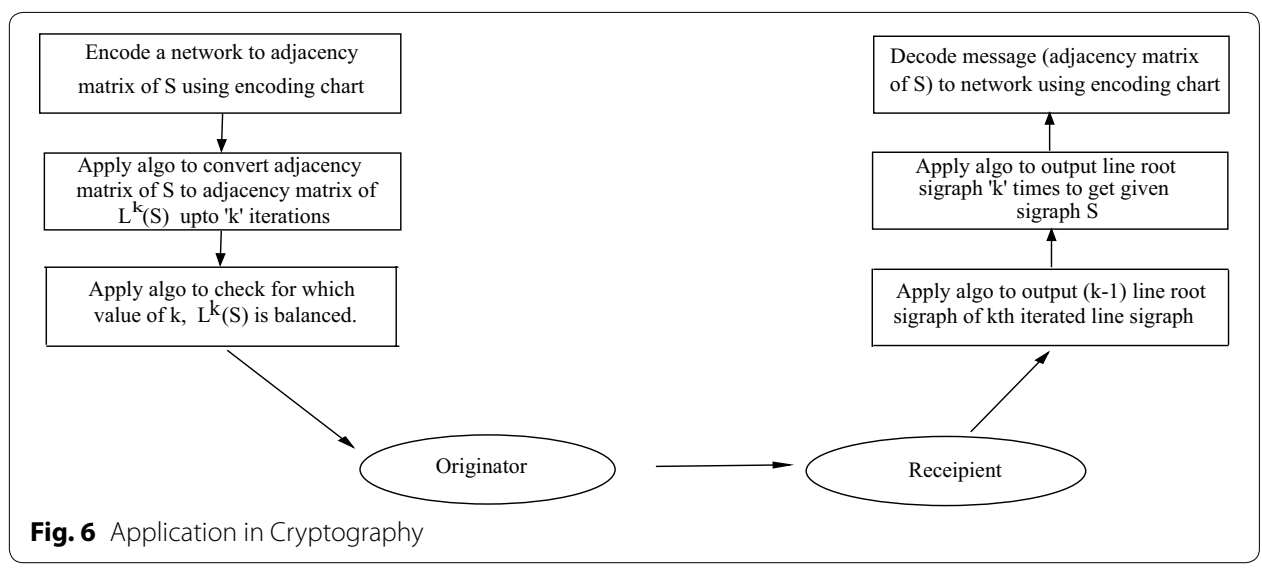




\section{Algorithm to detect a line sigraph and output its root sigraph}

The algorithm to detect a line sigraph and output its line root sigraph is an extension of a paper named "An Optimal Algorithm to Detect a Line Graph and Output its Root Graph" by Lehot (1974).

To check whether a given sigraph is a line root sigraph or not we have to check two conditions:

1. The underlying graph is a line graph.

2. Given sigraph is sign-compatible.

If both the conditions are satisfied we say that line root sigraph exists and will print the new line root sigraph of $S$.

Figure $7 \mathrm{a}$ shows the sigraph corresponding to the input matrix. Now we have to find the line root sigraph of this sigraph. The step wise procedure in shown in Fig. 7. NewNode represents the node of the intermediate graph, i.e., Fig. $7 \mathrm{~b}$ and Lookup represents if current index is mapped to which node. It shows the mapping between Fig. 7a, b. Since each node represents 2 points, maximum amount of numbers required is $2^{*}$ max where max denotes maximum value of $n$.

NewGraph denotes the adjacency matrix of required line root sigraph and corresponding to this matrix Fig. $7 \mathrm{c}$ is plot.

Find the first non-zero entry in adjacency matrix i.e. $\operatorname{graph}[i][j]$. For the first edge $(i, j)$ create a new node $(i, 1,2) \rightarrow$ Node $i$ named as pair $(1,2)$ i.e. in Fig. $7 \mathrm{~b}$.

Now start traversing the graph from this node. Also populate the final graph with this edge i.e. $(1,2)$. Now traverse through the graph and name other nodes. For each untraveled neighbor create a modified node for Fig. 7b. Find an $i 1$ for new node. We want to find $j$ as the first index $i 1$, we look if $j$ is assigned to any node, do we have any edge. For e.g. if $j=2$ and current nodes are $(a,(1,2)),(b,(2,3))$ and $(c,(1,4))$ we check if current node has edge from $a$ to $b$ Once we have find $j$ we want to find $k$ as second index. If $j$ and $k$ are found we have found the naming of $i 1$ and $i 2$. Add $i 1$ and $i 2$ to NewNode. Make CurIdx minimum of (CurId $x, j, k$ ). Record that $j$ and $k$ have been assigned to current node $(i)$.

If pairing of edges can be done as given by Lehot (1974), then we say that graph is a line graph otherwise not a line graph and hence not a line root sigraph.

This pair would represent an edge in NewGraph. If this node has negative edge anywhere, set this edge as negative else positive. Also add this node to the queue as we want to travel its neighbor.

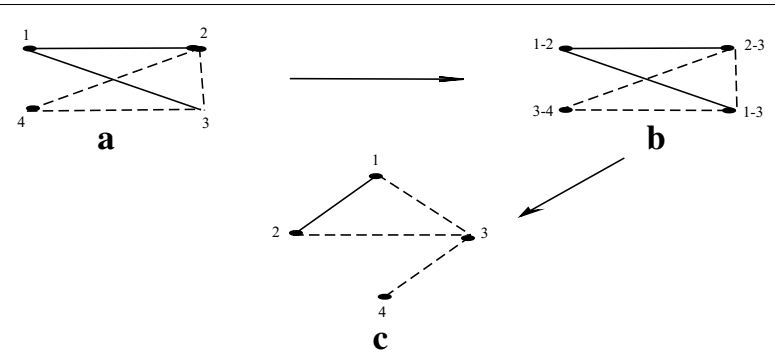

Fig. 7 Step wise procedure to find line root sigraph 
Next step we will check whether a given sigraph is sign-compatible or not. If sigraph is sign-compatible, line root sigraph exists and output the new modified graph otherwise line root sigraph does not exists.

Theorem 4 Sigraph $H$ is the line root of a sigraph $S$ and unique line root of a sigraph $S$ if and only

1. $S^{u}$ is a line graph and

2. Vertices of S can be assigned marks ' + ' or '-' such that both the ends of every negative edge receive '-' mark and the same is not true for any positive edge (i.e. $S$ is sign-compatible) such that the end vertices of the positive path receiving '-' mark is of length exactly two.

Theorem 5 Line root sigraph $H$ of a line sigraph $S$ is unique if and only if

1. H is homogenous and all-negative or

2. Positive section is of length one and every negative edge has at least one negative degree.

The final NewGraph is then plotted. This is the required line root sigraph. Since for a given line root sigraph we have many sigraphs, we have restricted ourselves to sigraphs based on Theorems 4 and 5 .

It uses following functions:

Max denotes maximum number of vertices

Structure of Node : idx; // Primary and i1, i2; // The pair represents mapping between Fig. 7a, b. Vertex 1 in Fig. 7a is mapped to pair 1-2 in Fig. 7b. idx represents initial vertex and $\mathrm{i} 1$ and $\mathrm{i} 2$ represents corresponding mapping between the vertices.

IsTraversed is a function which represents whether node is travelled or not.

Lookup defines if the current index is mapped to which node. Now, since each node is given 2 numbers, the maximum amount of numbers required is 2 MAX.

NewGraph represents required line root sigraph. 
Step 1. Input a sigraph $H$ with entries 0,1 and -1 whose line root sigraph $S$ is required.

Step 2. Find the first edge i.e repeat step for $i=0$ to Max and $j=0$ to Max and till queue is not empty.

(1) Check if $(\operatorname{graph}[\mathrm{i}][\mathrm{j}] !=0)$ If yes, then create a new node $(\mathrm{i}, 1,2)$. This node $\mathrm{i}$ is named as pair $(1,2)$. If no, goto Step 3.

(2) Again start traversing the graph from this node.

(3) Initialize weight $=1$.

(4) Repeat for $l=0$ to Max and weight $=1$ If true, assign weight $=-1$ else weight $=1$.

(5) Populate the final graph with this edge i.e $(1,2)$ NewGraph[NewNodes[i].i1][NewNodes[i].i2] = weight

NewGraph[NewNodes[i].i2][NewNodes[i].i1] = weight

Step 3. Now traverse through the graph and name other nodes. Traverse till queue is not empty.

3.1. Set current node to queue.front

3.2. Repeat for $\mathrm{i}=0$ to Max For each untravelled neighbor

3.2 .1 . create new node

3.2.2. set NewNode.idx $=$ current node i.e $\mathrm{i}$

3.2.3. Choose i1 and i2 such that NewGraph (adjacency matrix $=$ Lookup) and graph are consistent. First loop - Try with the indices tried until now

3.2.3.1. Look for i1 such that if (i1, l) is an edge, then (CurNode, l) should be an edge

3.2.3.2. Look for i2 such that if (i2, 1) is an edge, then (CurNode, l) should be an edge

3.2.3.3. Set CurIdx as the minimum of i1 and i2

3.2.3.4. Add edges to NewGraph from i1 and i2 to i. This pair would represent an edge in new graph. If this node has negative edge anywhere, set this edge as negative else positive.

3.2.3.5. Add the new node to the queue.

3.2.3.6. Set the current node as travelled. Second loop - If we go inside this loop, it means we did not find any i1 and i $<$ CurIdx. So we do an unbounded search. The steps are same, try with indices not use until now.

Step 4. Check if (CurIdx > Dim) then

Print Graph is not line graph and exit

else Print Graph is a line graph

Step 5. Since sigraph is a line graph now we have to check whether it is sign-compatible.

5.1. Set for $\mathrm{i}=1$ to $\mathrm{n}$ all counts to 0 .

5.2. Set for $\mathrm{i}=1$ to $\mathrm{n}$ and $\mathrm{j}=1$ to $\mathrm{n}$ and Count -1 in each row and column.

5.3. Set for $i=1$ to $n$ and $j=1$ to $n$

5.3.1. Search for first 1 and if(row $[\mathrm{i}] \geq 1$ and $(\operatorname{col}[\mathrm{j}] \geq 1)$,

If yes, Print "Sigraph is not sign-compatible" and exit else goto Step8.

Step 6. Print Sigraph is sign-compatible and hence its line root sigraph exists.

Step 7. Print the NewGraph (which is the required line root sigraph).

Step 8. Exit

\section{Complexity of computation involved in above algorithm}

In Step 2, we have to find first non-zero entry (i.e. first edge) in adjacency matrix of order $n \times n$ and then corresponding to each such entry, say $(i, j)$ th entry, we again have to traverse the graph to find its adjacent node and push the node in the queue.

Thus complexity of this step $=O\left(n^{3}\right)$.

In Step 3, since we have to traverse the graph and name all other nodes till queue is not empty. Queue contains the edges that are adjacent to first edge. This maximum number of edges is $e=n(n-1) / 2$. Thus complexity of this step $=O\left(e / n^{3}\right)$. 
In Step 5 we detect whether the given sigraph is sign-compatible or not. We count number of adjacent negative edges in adjacency matrix of order $n \times n$. Maximum number of edges can be $O\left(n^{2}\right)$. Thus complexity of this step $=O\left(n^{2}\right)$.

Total complexity $=O\left(n^{3}\right)+O\left(e / n^{3}\right)+O\left(n^{2}\right)=O\left(n^{3}\right)$.

Hence complexity of computation involved in above algorithm is $O\left(n^{3}\right)$, where $n$ is number of vertices in $S$ and $e$ is the number of edges.

\section{Encoding chart}

For a network with $n$ number of vertices, we have $n \times n$ adjacency matrix encoded as shown in Fig. 8.

$$
\left[\begin{array}{cccc}
0 & 1 & 0 & -1 \\
1 & 0 & -1 & -1 \\
0 & -1 & 0 & 1 \\
-1 & -1 & 1 & 0
\end{array}\right]
$$

\section{Encryption algorithm}

- Input the network which is to be encrypted. Then encode this network into adjacency matrix of $S$.

- Apply algorithm defined in Sinha and Sethi (2015) to convert a given adjacency matrix of $S$ to adjacency matrix of $L(S)$.

- Apply algorithm defined in Sect. 2.2.2 to detect if adjacency matrix of $L(S)$ so produced is balanced for $k=1$. If it is balanced, then the adjacency matrix of $L(S)$ is the encrypted data.

- Repeat algorithm defined in Sect. 2.2.1 to compute $k$ th iterated line sigraph and algorithm defined in Sect. 2.2.2 to detect its balancing at each iteration. Stop the procedure where we get iterated line sigraph which is balanced for some $k$.

- The adjacency matrix for which $L^{k}(S)$ is balanced for some ' $\mathrm{k}$ ' is the encrypted data.

- Now send the resultant adjacency matrix to the receiver in a linear format (i.e. either column wise or row wise) with space between elements. $n n<$ Resultant matrix data $>m m$ where, $n=$ number of vertices or nodes in the network $m=$ number of edges in the network.

\section{Decryption algorithm}

- Read the encrypted data and form the required matrices of order $m \times m$.

- Here the encrypted matrix is adjacency matrix of $L^{k}(S)$ which is balanced for some $k$.

- Apply "Algorithm to detect a line sigraph and output its root sigraph" defined in "Algorithm to detect a line sigraph and output its root sigraph" to obtain $L^{k-1}(S)$.

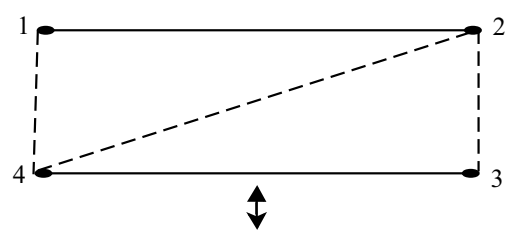

Fig. 8 Encoding of a network to adjacency matrix 
- Repeat the above step $(k-1)$ times to obtain back the resultant adjacency matrix of $S$.

- Decode adjacency matrix of $S$ using encoding chart to generate the original network.

Note: Since we have restricted adjacency matrix of $S$ to satisfy the property of $L^{k}(S)$ balancing for some ' $\mathrm{k}$ ', therefore, our networks are restricted to have data with values 0 , 1 and -1 and satisfying the balancing property of $L^{k}(S)$. The reading and writing of data can be done manually or by using file operation of any programming language( eg. $\mathrm{C}$, $\mathrm{C}++\ldots)$.

\section{Example}

Consider a network (Fig. 9):

Let

$$
A(S)=\left[\begin{array}{cccc}
0 & -1 & -1 & 0 \\
-1 & 0 & 0 & -1 \\
-1 & 0 & 0 & -1 \\
0 & -1 & -1 & 0
\end{array}\right]
$$

be the adjacency matrix for the above network.

Compute adjacency matrix of $L(S)$ corresponding to adjacency matrix of $S$ by applying algorithm defined in Sinha and Sethi (2015). Thus the resultant matrix is adjacency matrix of $L(S)$ :

$$
A L(S)=\left[\begin{array}{cccc}
0 & -1 & -1 & 0 \\
-1 & 0 & 0 & -1 \\
-1 & 0 & 0 & -1 \\
0 & -1 & -1 & 0
\end{array}\right]
$$

The corresponding graph is (Fig. 10):

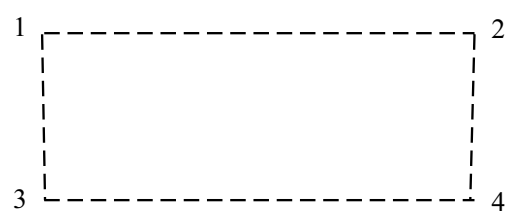

Fig. 9 Network

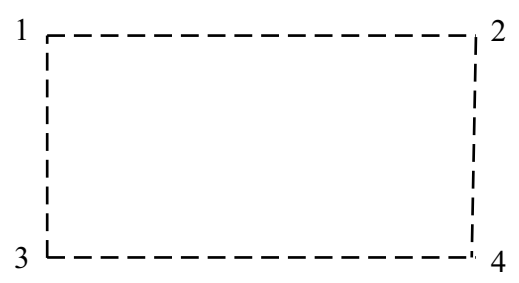

Fig. 10 Network corresponding to $L^{1}(S)$ 
Now we check whether this calculated $L^{1}(S)$ at $k=1$ is balanced or not. For this we apply algorithm defined in "Main Algorithm" from where it can be easily verified that it is balanced.

If we decide to transmit the data row wise, the data to be sent is ( the data are separated by space)

$440-1-10-100-1-100-10-1-1044$

Suppose the received data is

$440-1-10-100-1-100-10-1-1044$

From the data we have received we get the following matrix:

$$
T^{\prime}=\left[\begin{array}{cccc}
0 & -1 & -1 & 0 \\
-1 & 0 & 0 & -1 \\
-1 & 0 & 0 & -1 \\
0 & -1 & -1 & 0
\end{array}\right]
$$

isomorphic to some sigraph.

Now applying the decryption algorithm for $L^{k}(S)$ to $S$ as shown in example we get the matrix as

$$
T=\left[\begin{array}{cccc}
0 & -1 & -1 & 0 \\
-1 & 0 & 0 & -1 \\
-1 & 0 & 0 & -1 \\
0 & -1 & -1 & 0
\end{array}\right]
$$

which is equal to the adjacency matrix of $S$.

\section{Authors' contributions}

DS conceptualized this study and drafted the manuscript. The second author gave the shape to the manuscript by working out the algorithm and checking the complexity. All authors read and approved the final manuscript.

\section{Author details}

1 South Asian University Akbar Bhawan, Chanakyapuri, New Delhi 110 021, India. ${ }^{2}$ Center For Mathematical Sciences, Banasthali University, Banasthali 304 022, Rajasthan, India.

\section{Acknowledgements}

The authors express gratitude to Mr. Dhananjay Kulkarni who was always there in prior discussion and helping in writing algorithms and finding complexity and to the referees who made extensive and constructively critical comments on the first version of the paper.

\section{Competing interests}

Both authors declare that they have no competing interests.

Received: 12 September 2015 Accepted: 2 November 2015

Published online: 17 November 2015

\section{References}

Acharya BD (1981) A spectral criterion for cycle balance in networks. J Graph Theory 4(1):1-11

Acharya BD, Acharya M (1986) New algebraic models of a social system. Indian J Pure Appl Math 17(2):150-168

Acharya M, Sinha D (2006) Common-edge sigraphs. AKCE Int J Graphs Comb 3(2):115-130

Acharya M, Sinha D (2002) A characterizations of signed graphs that are switching equivalent to their jump signed graphs. Graph Theory Notes N Y XLIII:7-8

Acharya M, Sinha D (2003) A characterizations of sigraphs whose line sigraphs and jump sigraphs are switching equivalent. Graph Theory Notes N Y XLIV:30-34

Acharya M, Sinha D (2005) Characterizations of line sigraphs Nat Acad Sci Lett 28(1-2):31-34 [also, see extended

$$
\text { abstract in: Electron. Notes Discrete Math, } 15 \text { (2003)] }
$$

Behzad M, Chartrand GT (1969) Line coloring of signed graphs. Elem Math 24(3):49-52

Cartwright D, Harary F (1956) Structural balance: a generalization of Heider's theory. Psychol Rev 63:277-293

Chartrand GT (1977) Graphs as mathematical models. Prindle, Weber and Schmidt Inc, Boston 
Cormen T, Leiserson C, Rivest R, Stein C (2011) Introduction to algorithm, 3rd edn. PHI Learning Private Limited Deo N (1995) Graph theory with appliaction to Engineering and Computer Science. Prentice Hall India

Gill MK, Patwardhan GA (1981) A characterization of signed graphs which are switching equivalent to their line signed graphs. J Math Phys Sci 7(4):567-571

Gill MK, Patwardhan GA (1983) A characterization of signed graphs which are switching equivalent to their iterated line graphs. J Comb Inf Syst Sci 8:287-296

Golumbic MC (2004) Algorithmic graph theory and perfect graphs, 2nd edn

Harary F (1953) A characterization of balanced signed graphs. Mich Math J 2:143-146

Harary F (1969) Graph Theory. Addison-Wesley Publ. Comp, Reading

Harary F, Norman RZ, Cartwright DW (1965) Structural models: an introduction to the theory of directed graphs. Wiley Inter Science Inc, New York

Harary F, Kabell JA (1980/81) A simple algorithm to detect balance in signed graphs. Math Soc Sci 1:131-136

Horowitz E, Sahni S (2004) Computer Algorithm. Galgotia Publications

Lehot PGH (1974) An optimal algorithm to detect a line graph and output its root graph. J Assoc Comput Mach 21(4):569-575

Sinha D (2005) New frontiers in the theory of signed graph, Ph.D. Thesis, University of Delhi (Faculty of Technology)

Sinha D, Acharya M (2015) Characterization of signed graphs whose iterated signed line graphs are balanced or S-consistent. Bull Malays Math Sci Soc 28(1-2):31-34. doi:10.1007/s40840-015-0264-4

Sinha D, Sethi A (2015) An optimal algorithm to detect sign compatibility of a given sigraph. Nat Acad Sci Lett 38(3):235-238

Sinha D, Sethi A (2015) An algorithmic characterization of sigraphs whose common-edge sigraphs and second iterated line sigraphs are switching equivalent. J Discrete Math Sci Cryptogr 18(5):581-603. doi:10.1080/09720529.2015679

Sinha D, Sethi A (2015) An algorithm to detect S-consistency in line sigraph. J Comb Inf Syst Sci (Accepted)

West DB (1996) Introduction to graph theory. Prentice-Hall of India Pvt. Ltd

Zaslavsky T (1981) Characterizations of signed graphs. J Graph Theory 5:401-406

Zaslavsky T (1982) Signed graphs. Discrete Appl Math 4(1):47-74

\section{Submit your manuscript to a SpringerOpen ${ }^{\circ}$ journal and benefit from:}

- Convenient online submission

- Rigorous peer review

- Immediate publication on acceptance

- Open access: articles freely available online

- High visibility within the field

- Retaining the copyright to your article

Submit your next manuscript at $\mathbf{s p r i n g e r o p e n . c o m ~}$ 